\title{
From nano to micro and back
}

\author{
Working in large collaborations can help you understand how nanotechnology is closely related to other \\ fields, explains Elizabeth Huynh.
}

was an undergraduate student in Medical
and Health Physics when I was introduced
to nanotechnology, which for me at the
time was everything in science related to
nanoscale structures. During one summer,
I worked in Professor Gang Zheng's lab
at the University of Toronto where I was
intrigued by his approaches to image and
combat disease using small molecules
and nanoparticles. That summer, I
helped develop porphyrin nanoparticles
(Nature Mater. 10, 324-332; 2011) for
biophotonic imaging and therapy, and found
the experience exciting and meaningful, so
I decided to accept a position as a graduate
student in his lab.
My goal as a PhD student was to extend
the results obtained with porphyrin-based
nanoparticles to larger particles, with sizes in the micrometre range. The initial idea for a porphyrin microbubble was conceived through an international collaboration requiring a trimodal contrast agent for ultrasound, photoacoustic and fluorescence imaging. The contrast agent needed to have a micrometre-size gas core for ultrasound imaging and additional optical properties for photoacoustic and fluorescence imaging. The porphyrin-phospholipid building block developed in our lab met all the requirements for this type of multimodal contrast agent and I used it to synthesize porphyrin-based microbubbles. Although there was extensive expertise in our lab in developing porphyrin-based imaging and therapeutic agents, knowledge was lacking in the areas of microbubble characterization and ultrasound imaging. As I furthered our knowledge on microbubbles, I sought the advice of David E. Goertz, also at the University of Toronto, who is an expert in this field.

Initially, our microbubble contrast agent seemed to have nothing to do with nanotechnology. As the development of the trimodal contrast agent progressed, I questioned how this intrinsically multimodal porphyrin microbubble would respond after being destroyed by ultrasound, and hypothesized that there must be some remnant structure formed from the building blocks that would retain the porphyrin-related properties. This was the idea that resulted in the

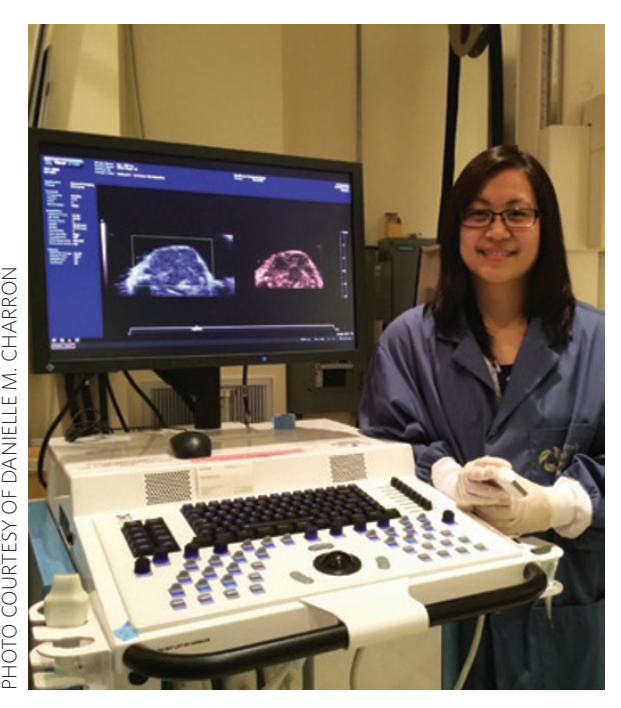

work on porphyrin nanoparticles being published on page 325 of this issue. In essence, we discovered that these porphyrin microbubbles could be triggered to form nanoparticles.

At the conception of this porphyrin microbubble project, I did not imagine that these microbubbles could be applied in the field of nanotechnology. However, our idea for a potential application came from our roots in nanotechnology and our understanding of the challenges of nanoparticle accumulation. This application of microtechnology for nanotechnology highlighted the notion that every field is, to some degree, interconnected and sometimes it is a matter of maintaining an open perspective to other fields throughout a project.

This study was a joint effort between researchers across the departments of medical biophysics, chemical engineering, and pharmaceutical sciences. For a successful collaboration with groups from different fields, open communication is key. We had to learn to speak each other's scientific language to explain the concepts and goals of the study, and to gain insight from a different field's perspective on the topic. For clear understanding and communication, I found that it was useful to have diagrams and analogies of concepts the collaborator was familiar with. Furthermore, initiative and persistence were also essential.
Although my study was important to the collaborators, they also had their own projects and other collaborations occupying their time and attention. Thus, it was important to follow-up regularly about the experiment and to discuss the expectations, not only with regard to the experimental design but also data analyses, timelines and manuscript contributions.

Although all the researchers were associated with the University of Toronto, we were scattered throughout the city, from a couple of blocks down the road to nearly a 30-minute drive away. One of the challenges I faced as a result of the distance was in translating the experimental expertise from one site, and attempting to replicate it and adapt it at another. For example, our study included an in vivo demonstration of the micro-to-nano phenomenon, which required multiple imaging modalities only available at our facility. However, one of our collaborators had the experience and a set-up for ultrasound microbubble animal experiments. Because of the different locations and the necessity to adapt their set-up for my own experiments, I had to recreate a similar set-up in our facility. This proved challenging and time consuming, as we needed to overcome the hurdles associated with setting up the equipment. What guided the success of this experiment were the consultations with our collaborators and that they helped to identify the problems and also helped brainstorm solutions to address them.

Interdisciplinary collaborations are an invaluable opportunity for researchers to step outside of their comfort zone and learn techniques unique to another field. Of even greater value is the opportunity to gain the perspective of researchers in different fields, such as how they approach problems, which is often different from our own. These lessons can broaden our understanding of nanotechnology and change the way we approach the challenges and limitations of our field.

\section{ELIZABETH HUYNH just completed} her PhD at the Department of Medical Biophysics, University of Toronto, Toronto, Ontario M5G 1L7, Canada.

e-mail: elizabeth.huynh@mail.utoronto.ca 